\title{
Radiolucent Foreign Body: A Diagnostic Dilemma
}

The Editor,

Sir,

Foreign bodies in the maxillofacial region are not unusual but are often overlooked. They may be missed due to their size, radiolucent nature, superimposition of bony structures and may pose diagnostic challenge with delayed treatment (1). Removal of the foreign bodies is delayed in approximately one-third of cases and removal has been reported even after 40 years $(2,3)$. Foreign bodies may lead to complications thereby causing morbidity, particularly in case of wood and porous mudstones which provide a good medium for the growth of microbial agents. The present case describes a radiolucent piece of clay pot at unusual facial sites and the challenges associated in its diagnosis and management.

A 55-year old male patient presented to hospital 25 days after a road traffic accident with stay sutures and purulent discharge on the face. Based on detailed history and thorough clinical examination, the foreign body was suspected to be road concrete, a sharp stone, wood or glass in the right midface region (Fig. 1).

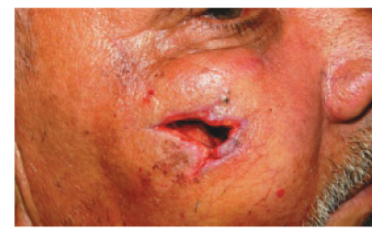

Fig. 1: Laceration wound in the cheek region.

There was no evidence of local and systemic causes for the unhealed wound. The simple radiographic examination revealed a hazy picture of foreign body which could be easily missed as it was present anterior to the right zygomatic buttress region overshadowing the right maxillary sinus (Fig. 2).

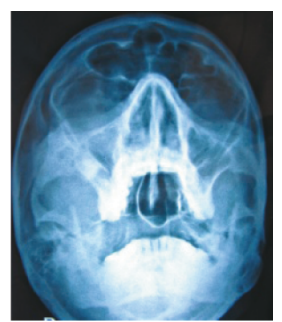

Fig. 2: Paranasal sinus radiograph shows foreign body in the right maxillary sinus region.
It was also suspected that the foreign body might be located in the maxillary sinus. Plain radiographs are usually the first investigation to be requested due to the low cost and easy access. However, radiographs in several planes or computed tomography scan should be considered for a high level of detection. Accurate localization before removal is essential. Blind searching is time consuming and may produce further trauma or displacement of foreign body into the deeper fascial planes.

In the present case, careful surgical explorations revealed unusual sharp pieces of clay pot approximately $4.5 \mathrm{x}$ $3.5 \mathrm{~cm}$ in partially formed fibrous capsule underneath the zygomatic buttress (Figs. 3 and 4).

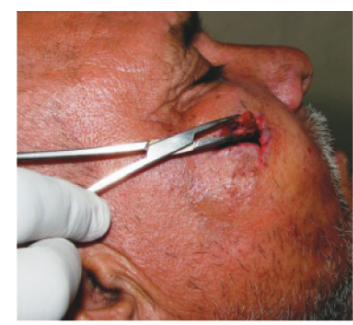

Fig. 3: Removal of the foreign body from laceration wound.

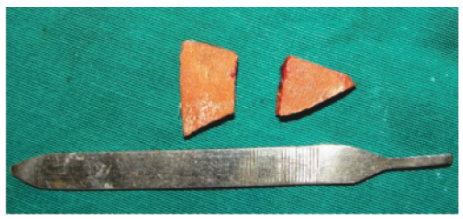

Fig. 4: Pieces of clay pot removed from the wound.

The maxillary sinus wall was intact and wound closure was done after thorough debridement. No complications were noticed during one-month follow-up. Since such wounds are contaminated, it is advisable to give tetanus and antibiotics prophylaxis before and after the surgery (4).

The penetrating wounds of the maxillofacial region appear deceptively minor and radiolucent foreign bodies could be easily missed. Thorough history, clinical examination and appropriate investigations are of utmost importance to prevent morbidity. Thus, it can be inferred that timely removal of foreign bodies can prevent significant morbidity to the patient.

Keywords: Foreign body, maxillofacial region, puncture wound 


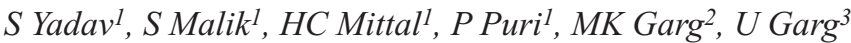
From: ${ }^{1}$ Department of Dental Surgery, ${ }^{2}$ Department of General Surgery and ${ }^{3}$ Department of Ear, Nose, and Throat, BPS Government Medical College for Women, Khanpur Kalan, Sonepat, Haryana, India.

Correspondence: $\operatorname{Dr} S$ Yadav, BPS Government Medical College for Women, Khanpur Kalan, Sonepat, Haryana, India. E-mail:dr.hiteshmittal@gmail.com

\section{REFERENCES}

1. Cameron M, Phillips B. Snookered! Facial infection secondary to occult foreign body. Int J Oral Maxillofac Surg 2006; 35: 373-5. Available from: http:/www.sciencedirect.com/science/article/pii/S0901502705003231

2. Robinson PD, Rajayogeswaran V, Orr R. Unlikely foreign bodies in unusual facial sites. Br J Oral Maxillofac Surg 1997; 35: 36-9.

3. Schnider N, Reichart PA, Bornstein MM. Intraoral foreign bodies detected 40 years after a car accident using cone beam computed tomography. Quintessence Int 2012; 43: 741-5. Available from: http:// qi.quitessenz.de/index.php? $\mathrm{doc}=\mathrm{abstract} \& \mathrm{abstractID}=28345$ /

4. Daya NP, Liversage HL. Penetrating stab wound injuries to the face SADJ 2004; 59: 55-9.

\section{Treatment of Attention Deficit Hyperactivity Disorder Accompanied by Epilepsy in a Child}

The Editor,

Sir,

Attention deficit hyperactivity disorder (ADHD) is a common neuropsychiatric disorder that occurs in childhood and goes on into adulthood. Atomoxetine is a potent, specific, norepinephrine reuptake inhibitor that has no other affinity on any other neuronal reuptake pumps used in the treatment of ADHD, alternatively $(1,2)$. High risks in terms of epileptic seizures have been observed in patients with $\operatorname{ADHD}(3,4)$. Here, we present the effect of atomoxetine on epilepsy in a boy with ADHD.

A 10-year old boy was admitted with complaints of negligence, short temper, inattention and academic failure in school. In his psychiatric examination, he was conscious, oriented and cooperated with a fluent speech. He had concentration problems, hyperactivity and impulsivity. There was no hallucination and delusion. His Wechsler Intelligence Scale for Children-Revised testing, laboratory results and physical examination were in the normal range. His condition was compatible with ADHD combined type according to the Diagnostic and Statistical Manual of Mental Disorders, fifth edition. He also had a history of epilepsy for four years. Valproic acid 750 $\mathrm{mg}$ /day has been used for three years. His last seizure had been two months ago, thus levetiracetam $600 \mathrm{mg} /$ day was added to his treatment. There was no treatment for psychiatry. Atomoxetine, $25 \mathrm{mg} /$ day, was initiated and the dose was titrated up to $40 \mathrm{mg}$ /day during the two weeks. In the first month of the follow-up period, the symptoms of attention deficiency decreased and school achievement improved. Furthermore, seizure was not observed in the subsequent year after the treatment of atomoxetine. Follow-up of the patient is ongoing and he tolerates the medication well.

Co-morbidity of epilepsy is a condition that requires attention in terms of treatment. Physicians should pay attention to the threshold of seizures. In the previous studies, the coexistence of ADHD and epilepsy was emphasized (3). In another study, evidence of increased risk of seizures related to stimulants was demonstrated (5). Although there is limited information about the treatment of ADHD accompanied with epilepsy by atomoxetine (6), based on our case, atomoxetine may be a safe treatment option in ADHD accompanied with epilepsy co-morbidity. However, this treatment option should be supported with further and well-attended multicentre studies.

Keywords: Attention deficit hyperactivity disorder, ADHD, children, epilepsy

N Yucel ${ }^{1}$, A Yucel $^{2}, \mathrm{H} \mathrm{Ozcan}^{2}$

From: ${ }^{1}$ Department of Child and Adolescent Psychiatry and ${ }^{2}$ Department of Psychiatry, Faculty of Medicine, Ataturk University, Erzurum, Turkey.

Correspondence: Dr A Yucel, Department of Psychiatry, Faculty of Medicine, Ataturk University, Erzurum 25040, Turkey. E-mail:dr_atakanyucel@hotmail.com

DOI: $10.7727 /$ wimj.2014.222

\section{REFERENCES}

1. Bymaster FP, Katner JS, Nelson DL, Hemrick-Luecke SK, Threlkeld PG, Heiligenstein JH et al. Atomoxetine increases extracellular levels of norepinephrine and dopamine in prefrontal cortex of rat: a potential mechanism for efficacy in attention deficit/hyperactivity disorder. Neuropsychopharmacology 2002; 27: 699-711.

2. Chalon SA, Desager JP, Desante KA, Frye RF, Witcher J, Long AJ et al. Effect of hepatic impairment on the pharmacokinetics of atomoxetine and its metabolites. Clin Pharmacol Ther 2003; 73: 178-91.

3. Davis SM, Katusic SK, Barbaresi WJ, Killian J, Weaver AL, Ottman R et al. Epilepsy in children with attention-deficit/hyperactivity disorder. Pediatr Neurol 2010; 42: 325-30.

4. McAfee AT, Holdridge KC, Johannes CB, Hornbuckle K, Walker AM The effect of pharmacotherapy for attention deficit hyperactivity disorder on risk of seizures in pediatric patients as assessed in an insurance claims database. Curr Drug Saf 2008; 3: 123-31.

5. Duplay D. Physician's Desk Reference. $59^{\text {th }}$ ed. Montvale, NJ: Thomson PDR; 2005.

6. Schubert R. Attention deficit disorder and epilepsy. Pediatr Neurol 2005; 32: $1-10$. 